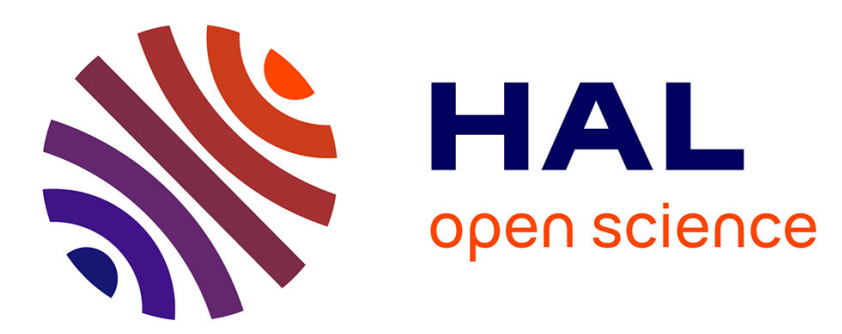

\title{
APPLICATION OF A SOOT FORMATION MODEL BASED ON AN INTERPOLATIVE CLOSURE METHOD OF MOMENTS TO A TURBULENT NON-PREMIXED FLAME
}

Sebastian Valencia, Cesar Celis, Luis Fernando Figueira da Silva

\section{To cite this version:}

Sebastian Valencia, Cesar Celis, Luis Fernando Figueira da Silva. APPLICATION OF A SOOT FORMATION MODEL BASED ON AN INTERPOLATIVE CLOSURE METHOD OF MOMENTS TO A TURBULENT NON-PREMIXED FLAME. 26th International Congress of Mechanical Engineering, Nov 2021, Florianopolis, Brazil. 10.26678/ABCM.COBEM2021.COB2021-0065 . hal-03357840

\author{
HAL Id: hal-03357840 \\ https://hal.science/hal-03357840
}

Submitted on 29 Sep 2021

HAL is a multi-disciplinary open access archive for the deposit and dissemination of scientific research documents, whether they are published or not. The documents may come from teaching and research institutions in France or abroad, or from public or private research centers.
L'archive ouverte pluridisciplinaire HAL, est destinée au dépôt et à la diffusion de documents scientifiques de niveau recherche, publiés ou non, émanant des établissements d'enseignement et de recherche français ou étrangers, des laboratoires publics ou privés. 


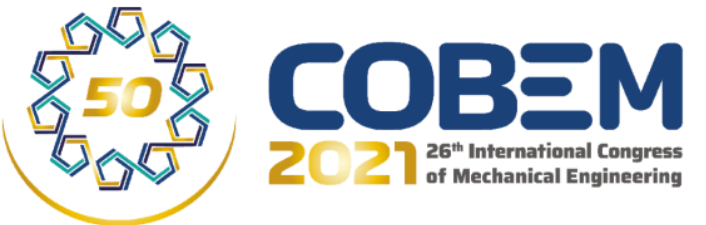

\section{COB-2021-0065 \\ APPLICATION OF A SOOT FORMATION MODEL BASED ON AN INTERPOLATIVE CLOSURE METHOD OF MOMENTS TO A TURBULENT NON-PREMIXED FLAME}

\section{Sebastian Valencia, Cesar Celis}

Mechanical Engineering Section, Pontificia Universidad Católica del Perú

Av. Universitaria 1801, San Miguel, Lima 32, Lima, Peru

svalenciar@pucp.edu.pe, ccelis@ @ucp.edu.pe

\section{Luís Fernando Figueira da Silva}

Deparment of Mechanical Engineering, Pontifícia Universidade Católica do Rio de Janeiro

Rua Marquês de São Vicente 225, Rio de Janeiro, RJ 22451-900, Brazil

luisfer@puc-rio.br

Abstract. The formation of soot in actual combustion systems involves several complex processes including those related to both soot chemical kinetics and soot particle dynamics. This means that detailed soot models are required to describe the formation of this critical pollutant in such systems. Accordingly, this work involves the development of a detailed soot formation model including its implementation in OpenFOAM. The particular method of moments-based modeling approach employed is the interpolative closure one (MOMIC), which uses interpolation and extrapolation to determine from first moments those higher, fractional, and negative order ones required in such modeling. More specifically, the transport equations for each of the first three (3) moments are solved, which in turn are compared with calculations carried out using a semi-empirical soot formation model (Brookes and Moss). These soot formation related comparisons are carried out accounting for turbulent non-premixed flames, and RANS as turbulence modeling approach, including soot nucleation, coagulation, surface growth and oxidation. For modeling combustion, the steady laminar flamelet model (SLFM) is used, considering the ABF as the chemical kinetic mechanism. Radiation effects are modeled in turn using the optically thin method. For nucleation, only acetylene is considered as a soot precursor in the two-equations model, whereas in the MOMIC one, benzene is accounted for. For soot oxidation, $O H$ and $O$ are considered as oxidant species in both models. The numerical results obtained here are compared with the corresponding experimental ones characterizing the Adelaide jet flame EHN (ethylene-hydrogen-nitrogen) 1, accounting for soot volume fraction and temperature profiles. The influence on soot predictions of soot models parameters are particularly discussed. When compared to the experimental data available in literature, the obtained numerical results present discrepancies in accordance with the limitations of the turbulence, combustion and soot modeling approaches employed here. It is expected that detailed soot models such the one developed in this work can be utilized in future to describe the formation of soot in practical combustion systems.

Keywords: Soot modeling, Method of Moments, Population Balance Equation, Turbulent diffusion flames, OpenFOAM

\section{INTRODUCTION}

Soot emissions are undesirable by-products generated in hydrocarbon fuels combustion processes characterized by fuel-rich mixtures and relatively high temperatures. This pollutant is harmful to both human health and environment, as well as it is an indicator of low combustion efficiency and excessive $\mathrm{CO}$ (carbon monoxide) and UHC (unburned hydrocarbons) emissions (Mueller and Raman, 2014). Over the years significant progresses have been made in deepening the understanding of soot formation in combustion processes. Nevertheless, due to the complexity of the interaction between the associated turbulent flow, gas phase chemical reactions, and the dynamics of soot particles, many crucial steps of this phenomenon, such as those present during the transition between gas phase PAHs (polycyclic aromatic hydrocarbons) and soot particle nucleation, are still not well understood (Lindstedt and Louloudi, 2005). Experimental data indicates that PAHs constitute the main precursors of soot particles (Mercier et al., 2019). Owing to the fact that the concentration of the largest molecular weight aromatics is not yet known with accuracy enough to be accounted for in soot formation processes indeed, for soot nucleation in recent studies only the smallest aromatics (A1 and A2) are accounted for (Gleason et al., 2021).

In order to achieve an accurate description, soot formation models must consider not only the chemical kinetics related to soot, but also implement mathematical algorithms capable of capturing the dynamics of the formed soot particles (Appel et al., 2000). However, due to the relatively large number of referred soot particles, tracking individual particles 
is challenging in soot formation modeling (Yang and Mueller, 2019). Therefore, statistical descriptions of the soot population particles evolution are often used in practice. Such descriptions involve solving a population balance equation (PBE) governing the evolution of the number density function (NDF) describing the concentration of soot particles of a given size (Gallen et al. 2019). Furthermore, since the chemical kinetics is strongly dependent on temperature, an adequate description of the associated radiation heat transfer is important. Under the presence of soot, peak temperatures can drop indeed by more than $300 \mathrm{~K}$ due to participating media radiation (Mehta et al., 2009). These phenomena are closely coupled and occur simultaneously in turbulent reacting flows.

The most recently developed soot formation models include those based on (i) the method of moments (MOM) (Frenklach, 2002; Mueller et al., 2009), where the moments of the particle size distribution function (PSDF) are solved; (ii) the sectional model (Netzell et al., 2007), where the PSDF is divided into discrete sections; and (iii) the Monte Carlo stochastic method (Balthasar and Kraft, 2003; Zhao et al., 2005), where no closure assumption is required (Wick et al., 2020). Due to the relatively high computational cost that the Monte Carlo method requires, which makes it unfeasible for multidimensional turbulent cases, both the method of moments and the sectional model are the most often applied approaches in such flames. Additionally, there are also simplified alternatives based on empirical correlations, such as the two-equation model (Brookes and Moss, 1999), that bring some benefits such as a low computational cost. These simple models are not capable however of dealing with the morphology of soot particles. In addition, the fact that some of these models have been developed for specific fuels only, methane for instance, represents an inherent limitation. In this work, due to its computational efficiency, numerical robustness and straightforward implementation in 3D CFD (computational fluid dynamics) simulations, the method of moments with interpolative closure (MOMIC) (Frenklach, 2002) has been utilized.

As described by Wick et al. (2020), there are two variants of MOMIC, (i) one where an additional negative moment is calculated in order to determine via interpolation the moments of negative fractional order, and (ii) another that uses only positive moments to determine the closure terms missing moments. Notice that a large number of moments is not strictly necessary to adequately describe soot formation. In fact, relatively good results have been obtained using three (3) moments in the past (Wick et al., 2020), reducing the associated computational cost. Accordingly, in this work soot formation models featuring three (3) moments have been implemented in the open source CFD software OpenFOAM. Additionally, the obtained results have been compared with others resulting from the use of a two equations semiempirical model (Brookes and Moss, 1999) available in the employed tool.

It is worth noticing that several measurement techniques often used for gas-phase flames are either not applicable in sooting flames or introduce unacceptably large errors (Mueller and Raman, 2013). Most of the experimental data available in literature includes thus measurements of temperature and soot volume fraction only, so soot model development is a challenge that must meet this shortcoming. In this work, the experimental data from the Adelaide sooting flame 1 (Mahmoud et al., 2018), including soot volume fraction axial and radial profiles and temperature axial profile, are used for comparison purposes with the implemented soot models. This particular flame is a turbulent diffusion jet one involving ethylene/hydrogen/nitrogen as fuel mixture. In the Adelaide sooting flame 1 related experiment, ethylene was chosen as fuel due to its high soot yield and relatively well-established chemical kinetics, whereas $\mathrm{N}_{2}$ was used to lower the concentration of soot in the flame and $\mathrm{H}_{2}$ to maintain the flame attached to the burner (Sun et al., 2017). Accordingly, this work is structured as follows. The mathematical background including the combustion, radiation and soot formation models are described in Section 2. In Section 3 in turn, the soot model implementation in OpenFOAM is introduced. In Section 4, the studied test case for model analysis and verification is discussed. The main results, including a thorough analysis of the performance of the soot models implemented, are presented and discussed in Section 5. Finally, Section 6 summarizes the main conclusions drawn from the obtained results.

\section{MATHEMATICAL MODELING}

\subsection{Governing equations}

Low Mach number turbulent flows with variable density are accounted for in this work. Accordingly, the Reynolds Average Navier-Stokes modeling approach (RANS) is used together with the standard $k-\varepsilon$ turbulence model (Launder and Sharma, 1974). The numerical approach employed here is based on time averaging of the flow governing equations related to mass, momentum and energy. In this work, two additional transport equations are solved to compute the turbulent kinetic energy $(k)$ and the turbulent dissipation rate $(\varepsilon)$. RANS has been used here because of its lower computational cost when compared to other more expensive approaches such as LES (large eddy simulation) and DNS (direct numerical simulation). Notice that when dealing with model development, involving tens or even hundreds of numerical simulations, computational cost is a critical issue.

\subsection{Combustion modeling}

The combustion model employed here is the steady laminar flamelet (SLF) (Peters,1984) one, which accounts for a turbulent flame as an ensembled of thin one-dimensional flamelets within the turbulent flow field. In this model 
temperature and species mass fractions values are stored in lookup tables in terms of mixture fraction, $Z$, and scalar dissipation rate at stoichiometric conditions, $\chi_{s t}$. The advantage of this model is that the chemistry involved can be preprocessed and tabulated, significantly reducing thus the computational cost associated with the modeling of turbulent flames. The SLF model however is not able to properly describe combustion processes involving fast chemistry, and it cannot capture deep non-equilibrium effects such as ignition, extinction and slow chemistry (Pierce and Moin, 2004).

\subsection{Radiation modeling}

In terms of radiation energy transport, the optically thin radiation model (Guo and Smallwood, 2004) is utilized in this work. In this simplified approach often used in combustion calculations, only radiation emission is accounted for and reabsorption is neglected. This method has enabled the prediction of the behavior of several laboratory flames, but it is known to overestimate radiation effects (Cuoci et al., 2008). In this method, in the energy transport equation, radiation is accounted for as a source heat transfer term,

$$
Q_{r}=-4 \sigma a\left(T^{4}-T_{e n v}{ }^{4}\right) \text {, }
$$

where $\sigma$ is the Stefan-Boltzmann constant, $a$ is the Planck-mean absorption coefficient of the gas mixture and $T_{e n v}$ is the environment temperature. Indeed, $\mathrm{a}$ is a contribution of $\mathrm{CO}, \mathrm{H}_{2} \mathrm{O}, \mathrm{CO}_{2}, \mathrm{CH}_{4}$ and soot, according to:

$$
a=p_{\mathrm{H}_{2} \mathrm{O}} a_{p, \mathrm{H}_{2} \mathrm{O}}+p_{\mathrm{CO}_{2}} a_{p, \mathrm{CO}_{2}}+p_{\mathrm{CO}} a_{p, \mathrm{CO}}+p_{\mathrm{CH}_{4}} a_{p, \mathrm{CH}_{4}}+f_{v} a_{\text {soot }},
$$

where $p_{i}$ is the partial pressure of chemical species $i$, and $f_{v}$ is the soot volume fraction. As highlighted in Eq. (3) for $\mathrm{H}_{2} O$ and $\mathrm{CO}_{2}$, and in Eq. (4) for $\mathrm{CO}$ and $\mathrm{CH}_{4}, a_{p, i}$ are functions of temperature and the extinction coefficients $c_{j}, j=1, \ldots, 5$.

$$
\begin{aligned}
& a_{p}=c_{0}+c_{1}\left(\frac{1000}{T}\right)+c_{2}\left(\frac{1000}{T}\right)^{2}+c_{3}\left(\frac{1000}{T}\right)^{3}+c_{4}\left(\frac{1000}{T}\right)^{4}+c_{5}\left(\frac{1000}{T}\right)^{5} \\
& a_{p}=c_{0}+c_{1} T+c_{2} T^{2}+c_{3} T^{3}+c_{4} T^{4}
\end{aligned}
$$

For the gaseous species in this work, the extinction coefficients are taken from Barlow et al. (1998), whereas the soot related extinction coefficient is evaluated in turn using,

$$
a_{\text {soot }}=1232 \rho_{\text {soot }}\left[1+4.8 * 10^{-4}(T-2000)\right]
$$

\subsection{Soot modeling}

In this work, two soot formation models will be developed, the two-equations semi-empirical one by Brookes and Moss (1999) and the detailed soot formation model MOMIC (method of moments with interpolative closure) (Frenklach, 2002).

\subsubsection{Two-equations model}

In this semi-empirical model, soot is calculated from solving transport equations for soot mass fraction $Y_{s}$, and normalized soot nuclei concentration $b_{n u c}$ (Brookes and Moss, 1999). A thermophoretic diffusivity term has been added to the original soot mass fraction transport equation, Eq. (6), due to both soot is mainly transported by temperature gradients and molecular diffusivity related effects can be neglected (Cuoci et al., 2008). In this work however the molecular diffusivity term has been maintained to ensure the model numerical stability.

$$
\begin{aligned}
& \frac{\partial \rho Y_{s}}{\partial t}+\nabla \cdot(\rho v) Y_{s}=\nabla \cdot\left(\frac{\mu_{t}}{\sigma_{s}} \nabla Y_{s}\right)+\nabla \cdot\left(\rho V_{t}\right) Y_{s}+\frac{d M}{d t}, \\
& \frac{\partial \rho b_{n u c}}{\partial t}+\nabla \cdot(\rho v) b_{n u c}=\nabla \cdot\left(\frac{\mu_{t}}{\sigma_{\text {nuc }}} \nabla b_{n u c}\right)+\nabla \cdot\left(\rho V_{t}\right) b_{n u c}+\frac{1}{N_{n o r m}} \frac{d N}{d t} .
\end{aligned}
$$

In Eqs. (6) and (7), $M$ and $N$ stands for soot mass concentration and soot particle number density, respectively. $\sigma_{s}$ is in turn the Prandtl number, whereas $\rho, v$ and $\mu$ represent, respectively, reacting mixture density, velocity and viscosity, whereas the thermophoretic velocity is defined as $V_{t}=-\frac{0.55 v}{T} \nabla T$. The rate of nuclei concentration $d N / d t$ and the rate of mass fraction $d M / d t$, Eq. (8) and (9), respectively, are source terms used to model the contribution to soot formation of nucleation, coagulation, surface growth and oxidation.

$$
\frac{d N}{d t}=\left(\frac{d N}{d t}\right)_{\text {nucleation }}-\left(\frac{d N}{d t}\right)_{\text {coagulation }} \text {, }
$$




$$
\frac{d M}{d t}=\left(\frac{d M}{d t}\right)_{\text {nucleation }}+\left(\frac{d M}{d t}\right)_{\text {surface growth }}-\left(\frac{d M}{d t}\right)_{\text {oxidation }},
$$

In this work, the nucleation rate model employed is the one proposed by Brookes and Moos (1999), which models soot nucleation by a simple one-step reaction of acetylene. The oxidation rate model utilized here is in turn the one developed by Lee et al. (1980), where $\mathrm{OH}$ and $\mathrm{O}_{2}$ are considered as oxidant species for soot. Each of the terms appearing in Eq. (8) and (9) are obtained through empirical correlations, whose reaction rate parameters and scaling factors are available in Brookes and Moss (1999).

\subsubsection{Method of moments}

In order to model soot formation, it is necessary to describe the particle size distribution function (PSDF), but this would require solving the population balance equation for an infinite number of size classes. One particular approach frequently used to describe the PSDF is the method of moments, where a small number of moments is used only. When applied to soot modeling, the method of moments has many variants. In this work indeed, due to its computational efficiency and its numerical robustness, the focus is on the method of moments with interpolative closure (MOMIC) one (Frenklach, 2002; Wick et al., 2020). In MOMIC, the PSDF is described by a finite number of lower-order moments only. In particular, the $r$-th moment of the particle number density $N_{i}$, and the reduced moment $\mu_{r}$, are (Balthasar and Frenklach, 2005),

$$
\begin{aligned}
& M_{r}=\sum_{i=1}^{\infty} m_{i}^{r} N_{i}, \\
& \mu_{r}=\frac{M_{r}}{M_{0}}
\end{aligned}
$$

where $m_{i}$ and $N_{i}$ stands for, respectively, the mass and number density of particles of size class $i$. The zero-order moment $M_{0}$ and the first-order one $M_{1}$ have physical interpretations here. $M_{0}$ is the total particle number density and $M_{1}$, when divided by the soot density, represents the soot volume fraction. Accordingly, the governing equation for the $r$-th moment is given by,

$$
\frac{\partial\left(\rho M_{r}\right)}{\partial t}+\nabla \cdot\left(\rho v M_{r}\right)=\nabla \cdot\left(\frac{\mu_{e f f}}{\sigma_{t}} \nabla M_{r}\right)+\nabla \cdot\left(\rho V_{t}\right) M_{r}+\frac{\partial M_{r}}{\partial t},
$$

where $\mu_{e f f}$ is the effective diffusion coefficient, $\sigma_{t}$ is the turbulent Prandtl number, and $\partial M_{r} / \partial t$ is the moment transport equation source terms, which contain the contributions from soot particle nucleation, coagulation and heterogeneous reactions with gas-phase species. Notice that the molecular diffusivity term was also accounted for in this model to guarantee its numerical stability A general form of these source terms can be written as (Frenklach, 2002),

$$
\frac{\partial M_{r}}{\partial t}=R_{r}+G_{r}+W_{r}
$$

where $R_{r}, G_{r}$ and $W_{r}$ are the source terms due to nucleation, coagulation and surface growth (including oxidation). Mathematical descriptions for each of these source terms are found in (Kazakov and Frenklach, 1998; Appel et al., 2000; Mehta et al., 2009).

Soot nucleation is usually modeled as coagulation between two PAH molecules. In this work, due to both its simplicity and the fact that it does not need additional moments equations as other models do so (Frenklach and Wang, 1994), the formulation proposed by Rezvan et al. (2002) is utilized. For the sake of simplicity, it is assumed that only the smallest aromatics are involved in soot formation (Gleason et al. 2012), thus the one-ring PAH (benzene) is considered here as soot precursor specie.

Coagulation is the process in which soot particles collide with each other to form larger spherical particles. However, after a certain soot particles size, these particles no longer coalesce but form chains of soot aggregates. This last phenomenon is called soot aggregation and it will not be modeled in this work. The coagulation phenomenon is based on the Smoluchowski equations (Smoluchowski, 1917), where the collision coefficient $(\beta)$ is described for all coagulation regimes, free molecular, continuum and transition. These regimes are classified as a function of the Knudsen number, $k_{n}=\frac{\lambda}{d_{s}}$, where $\lambda$ is the gas mean free path and $d_{s}$ is the soot particle diameter (Kazakov and Frenklach, 1998).

Surface growth and oxidation are modeled in this work using the hydrogen abstraction $\mathrm{C}_{2} \mathrm{H}_{2}$ addition mechanism (HACA) (Frenklach and wang, 1994). In the HACA mechanism, the reactions rates are computed using Arrhenius form equations employing the rate coefficients summarized by Appel et al. (2000).

In order to solve the closure problem of the coagulation source term $G_{r}$, it is necessary to know the reduced moments of fractional and negative order present in the $G_{r}$ formulation. In MOMIC, this is carried out through Lagrange 
interpolation, obtaining high-order, negative-order and fractional-order moments from the first low-order moments (Frenklach, 2002). Wick et al. (2020) emphasize that the highest interpolation accuracy is obtained using two interpolation schemes, (i) one for the positive moments (obtaining higher accuracy with higher number of moments), and (ii) the other for the negative ones (using infinite minus moments in the interpolation among $M_{-\infty}, M_{0}$ and $M_{1}$ ) (Wick et al., 2020). In this work, the positive-order fractional moments are computed from all integer moments solved in the transport equations as,

$$
\log \left(\mu_{p}\right)=L_{p}\left(\log \left(\mu_{0}\right), \log \left(\mu_{1}\right), \ldots . ., \log \left(\mu_{r \max }\right)\right),
$$

where $L_{p}$ is the Lagrange interpolation function, $p$ stands for the order of the reduced moment $\mu_{p}$ to be solved, and $\mu_{r \text { max }}$ is the maximum integer moment solved in the transport equations. Similarly, the negative-order fractional moments are solved considering just the first three moments, as it yields reasonably accurate results similar to the case when using an infinite minus moment (Frenklach, 2002). The Lagrange extrapolation function for the negative-order fractional moments utilized here is,

$$
\log \left(\mu_{p}\right)=L_{p}\left(\log \left(\mu_{0}\right), \log \left(\mu_{1}\right), \log \left(\mu_{2}\right)\right)
$$

In the free molecular regime, due to the non-additive character of the collisions coefficients $(\beta)$, a grid function $f_{l}^{x, y}$ is employed. As proposed by Frenklach (2002), a double interpolation scheme is required to evaluate soot coagulation under this regime. The first interpolation is employed to obtain the value of the fractional order grid function from integer order grid functions as,

$$
\left(f_{1 / 2}^{x, y}\right)=L_{1 / 2}\left(\log \left(f_{0}^{x, y}\right), \log \left(f_{1}^{x, y}\right), \log \left(f_{2}^{x, y}\right)\right),
$$

where $L_{1 / 2}$ indicates the Lagrange interpolation polynomial, and the second interpolation is used to determine, from the integer moments using Eq. (14) and Eq. (15), the fractional order moments.

\section{OPENFOAM IMPLEMENTATION}

The numerical solver aiming to model soot formation in turbulent diffusion flames has been developed using the open source CFD tool OpenFOAM (OpenCFD, 2021). The associated C++ based computational code has been implemented using the OpenFoam 7.0 libraries. Both the two-equation model and the MOMIC model were initially coupled as postprocessing tools. Due to the importance of heat radiation transfer in sooting flames and in order to have a soot-temperature coupling, these soot models were subsequently coupled with a steady-state flamelet-based solver focused on turbulent diffusion flames called flameletSimpleFoam (Holtzmann, 2021).

In order to implement the MOMIC model in OpenFoam, Eq. (12) was added to the flamelet solver, for each transported moment. These added equations along with the transport ones for mass, momentum, turbulent kinetic energy, turbulent dissipation rate, enthalpy, mean mixture fraction, and mixture fraction variance form the set of equations solved here. In particular, the new transported moments related equations were written as an object of the OpenFOAM class, fvScalarMatrix, using the mathematical notations available in OpenFOAM in terms of divergence, gradient, Laplacian and time derivatives.

More specifically, the moments equations implementation was carried out by declaring and defining each of the moments in the file named createFields. H. The source terms for each moment were defined as in Eq. (13) using the nucleation, coagulation, surface growth/oxidation schemes described above. Three interpolation functions were implemented, for the positive fractional order moments and for the negative fractional order, and one for the grid function. Additionally, in order to couple the radiation heat transfer to the solver, the energy equation, HEqn.H, was modified. The soot radiation source term defined in Eq. (5) was added to the Qrad term present in the solver. It is worth noticing that the flameletSimpleFoam solver is a solver for non-adiabatic flames, so in order to consider the enthalpy variation due to radiation a new term called enthalpy defect was accounted for.

The gradient schemes for each transported moment are cellLimited Gauss Linear, the divergent schemes are bounded gauss limited linear and the Laplacian schemes are Gauss linear corrected. The solver employed for each term is the $P B i C G$ with a tolerance of $10^{-8}$. The relaxation factor for the first moment is 0.2 , and for the rest of the moments a factor of 0.5 was employed. These values were defined through trial-and-error tests based on convergence attainment.

The numerical verification of the MOMIC model implemented here was carried out by means of comparisons with results obtained using the commercial software ANSYS Fluent (ANSYS, 2021). For these comparisons, both solvers employed the same kinetic mechanism, as well as the same turbulence modeling approach. In both tools the SLFM with flamelets created in OpenFOAM was utilized. When comparing the transported moments computed values, discrepancies of about $5 \%$ or lower were obtained. For the sake of brevity these numerical verification related results are not shown here. 


\section{CASE STUDY AND SIMULATION CONFIGURATION}

\subsection{Case study}

In order to validate the soot formation models implemented in this work, the Adelaide jet flame EHN (ethylenehydrogen-nitrogen) 1 (Mahmoud et al., 2018) was utilized as test case. As its name indicates, this is an ethylene, hydrogen and nitrogen (EHN) jet flame with a jet Reynolds number of 15000 . The molar concentration of the fuel mixture is (39.1/41/19.9) \% at $294 \mathrm{~K}$ and $1 \mathrm{bar}$, whereas the total mixture density is $0.717 \mathrm{~kg} / \mathrm{m}^{3}$ and the dynamic viscosity 1.215 $10^{-5} \mathrm{~kg} /(\mathrm{m} . \mathrm{s})$. The mean flow jet velocity is $56.8 \mathrm{~m} / \mathrm{s}$, which is surrounded by a co-flow air stream with an average velocity of $1.1 \mathrm{~m} / \mathrm{s}$ and turbulent intensity of $1.5 \%$ at $294 \mathrm{~K}$. The studied configuration consists of a straight pipe jet burner made of Aluminum with $4.4 \mathrm{~mm}$ of inner diameter, $1 \mathrm{~mm}$ of wall thickness and a length of $385 \mathrm{~mm}$. The co-flow duct is a square-cross section of 150x150 mm and the jet pipe outlet rises above the duct up to a distance of $18 \mathrm{~mm}$. The pipe is tapered as it is shown in the Figure 1a. The available experimental measurements are axial and radial soot volume fraction profiles and axial temperature profile. Temperature measurements were made with R-type thermocouple of $1 \mathrm{~mm}$ bead diameter with $4 \%$ uncertainty, whereas Laser Induced Incandescence (LII) was used to measure soot volume fraction, with uncertainties of about $25 \%$.

\subsection{Simulation configuration}

A three-dimensional wedge-shaped computational domain, discretized using the OpenFOAM utility blockMesh, was accounted for. An O-grid mesh is employed to simulate the symmetrical flame with a wedge angle of $5^{\circ}$ and wedge type boundary conditions on both sides. The computational domain dimensions are $870 \mathrm{~mm}$ on the z-axis and $75 \mathrm{~mm}$ on the $\mathrm{X}$-axis. In addition, the duct length is $70 \mathrm{~mm}$. As it may be seen in Figure 1b, in order to properly describe flow properties related gradients, the mesh is more refined in the domain region close to the fuel jet. In order to save computational time in turn, in both the co-flow region and the flame further zone the mesh elements are coarser. The computational mesh employed features 33200 elements. The smallest element has a characteristic size of $125 \mu \mathrm{m}$ and it is located in the jet fuel outlet zone and on the inner wall of the jet pipe near to the jet exit, which ensures that $y+<4$. The largest element features in turn a characteristic size of $10 \mathrm{~mm}$ at the outlet and radial region farthest from the axis.

a)

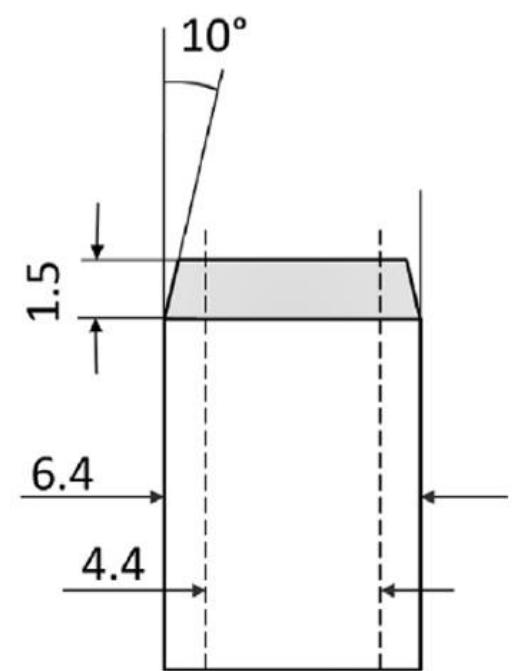

b)

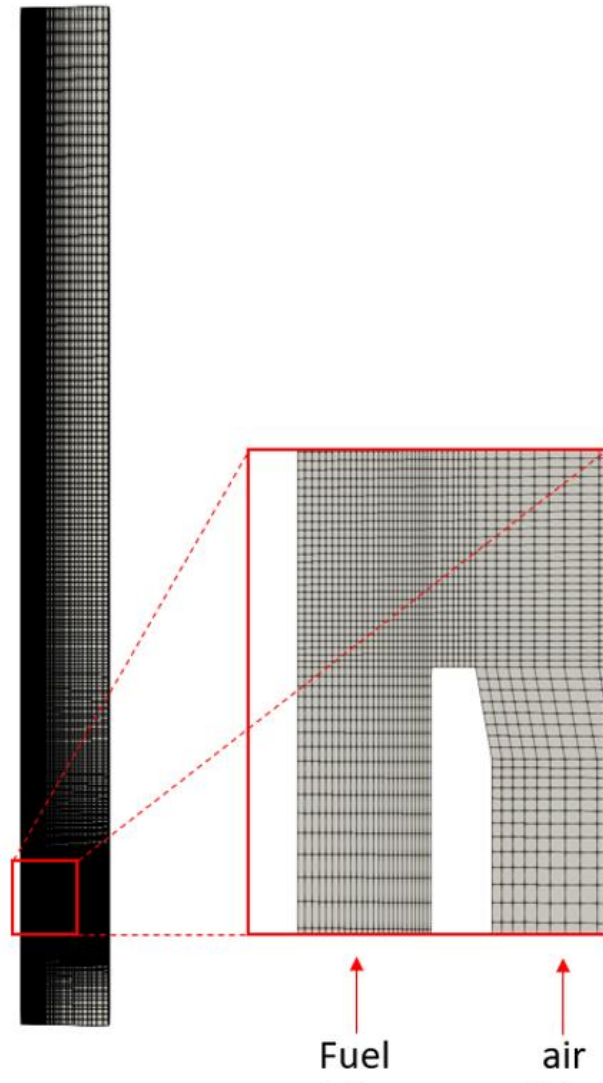

inlet inlet

Figure 1. a) Tapered section of pipe jet burner (Mahmoud et al., 2018). b) Computational mesh utilized. 
In order to compute mesh-independent results, a mesh independence study was carried out as well employing grids featuring 12200, 22300 and 33200 elements. In particular, temperature axial profiles and mass fractions ones associated with the main soot precursor species were analyzed for each mesh. Accordingly, Figure 2 a shows the mass fraction axial profiles of $\mathrm{C}_{2} \mathrm{H}_{2}$ and $\mathrm{OH}$, whereas Figure $2 \mathrm{~b}$ does so for chemical species $\mathrm{C}_{6} \mathrm{H}_{6}$ and $\mathrm{O}_{2}$. From Figure 2 results, it is noticed that when using 22300 mesh elements or more the associated results no longer change. In the case of temperature, the discrepancies among the profiles obtained with the referred three meshes were negligible indeed so they are not shown here. Therefore, a computational mesh featuring 33200 elements was employed here.
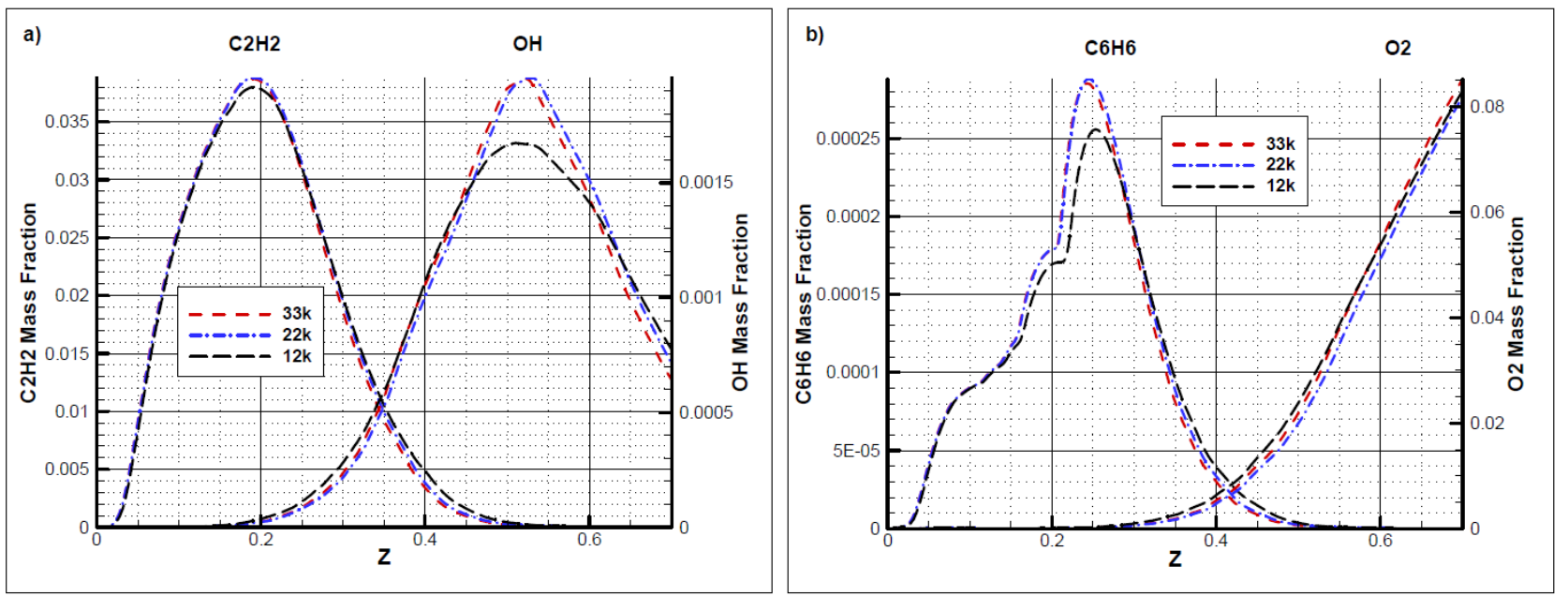

Figure 2. a) Mass fractions axial profiles of $\mathrm{C} 2 \mathrm{H} 2$ and $\mathrm{OH}$. b) Mass fractions axial profiles of C6H6 and $\mathrm{O} 2$.

In terms of boundary conditions, a temperature of $294 \mathrm{~K}$ and a pressure of $101.325 \mathrm{kPa}$ were accounted for at both fuel and air inlets. The considered fuel inlet velocity is $56.8 \mathrm{~m} / \mathrm{s}$, whereas the co-flow air one is $1.1 \mathrm{~m} / \mathrm{s}$. Turbulent boundary conditions were estimated from the turbulent intensity of $5 \%$ on the fuel side and $1.5 \%$ on the air side, obtaining a turbulent kinetic energy value of $11,149 \mathrm{~m}^{2} / \mathrm{s}^{2}$ for the fuel inlet and $4.0810^{-4} \mathrm{~m}^{2} / \mathrm{s}^{2}$ for the air one. In a similar fashion, turbulent dissipation rate values accounted for include $19679 \mathrm{~m}^{2} / \mathrm{s}^{3}$ for the fuel inlet and $7.0710^{-5} \mathrm{~m}^{2} / \mathrm{s}^{3}$ for the air one, respectively. In the case of velocity, the outlet is specified as a pressureInletOuletVelocity boundary condition, which implies a zero-gradient pressure condition in the outflow, and the burner wall is specified as a noSlip one, which fixes the velocity to zero at walls. For pressure, the zerogradient condition was set for the inlets and walls, whereas the outlet was specified with a totalPressure type boundary condition with a value of $101.325 \mathrm{kPa}$. In the case of $k$, the kLowReWallFunction condition was specified on the burner wall, which provides a wall constraint on the turbulent kinetic energy for low and high Reynolds number turbulence models, whereas zerogradient was enforced on the domain wall. In the case of epsilon, the epsilonWallFunction condition was used for the burner wall, which provides a wall constraint on the turbulent kinetic dissipation rate for low and high Reynolds number turbulence models, whereas zerogradient was imposed on the domain wall. Mean mixture fraction was set to 1 in the fuel inlet and 0 in the air inlet, whereas the mixture fraction variance was set to 0 in both inputs. For both $\mathrm{Z}$ and $\mathrm{Zvar}$, the zerogradient condition was set for all walls. The flamelets used here were created from the gas-phase chemical kinetic mechanism ABF (Appel et al., 2000) using the flamelet generator included in the flameletSimpleFoam solver. The ABF mechanism contains 101 chemical species and 543 reactions, and the larger $\mathrm{PAH}$ accounted for is pyrene $\left(\mathrm{C}_{16} \mathrm{H}_{10}\right)$.

\section{RESULTS AND DISCUSSION}

The main numerical results obtained in this work are discussed in this section.

\subsection{Temperature and Soot volume fraction results}

Figure 3 shows the MOMIC based mean temperature profile and the soot volume fractions computed with both MOMIC and the two-equations soot model employed here. All results shown in this figure include radiation effects. In qualitative terms, Figure 3 shows a distribution of soot in accordance with the type of fuel used and the structure of the flame accounted for. For the temperature profile (Figure 3a), the optically thin radiation model and the MOMIC soot model was considered. From the soot volume fraction predictions carried using both soot models, soot appears in the same physical region and the soot maximum peak is located at around $0.3 \mathrm{~m}$ above the exit jet. In quantitative terms however, soot volume fraction related discrepancies between numerical predictions and experimental data exist. The potential sources of these discrepancies will be discussed in the following sections. 


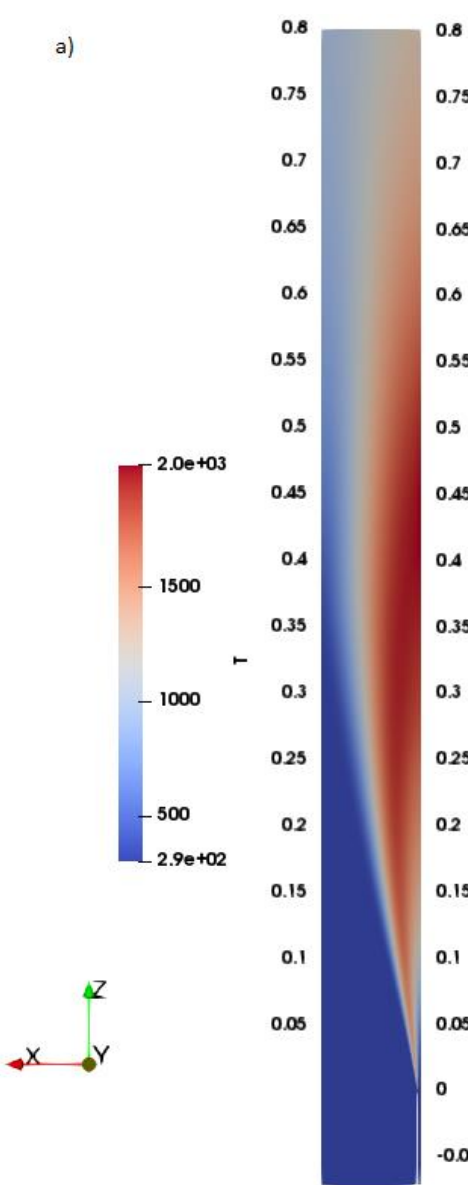

b)

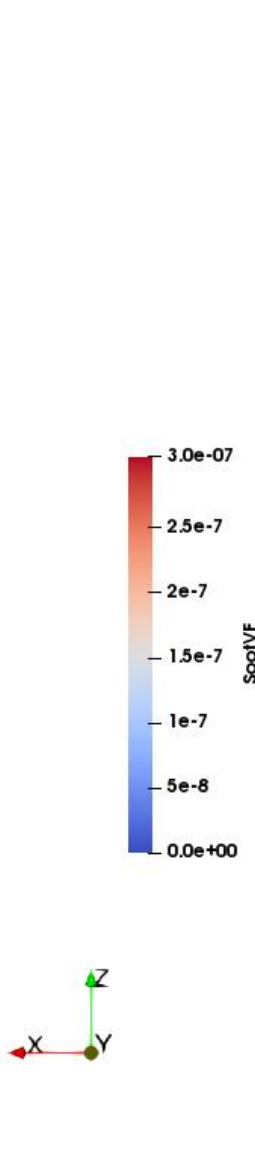

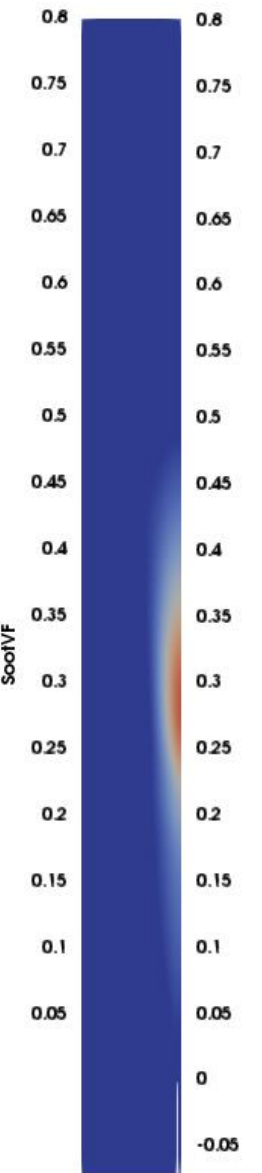

c)

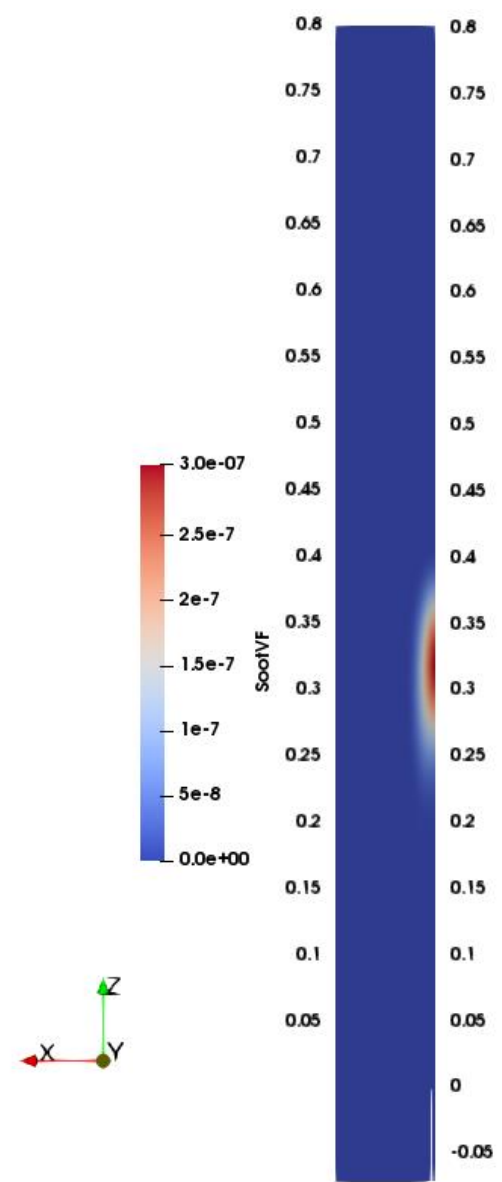

Figure 3. a) MOMIC mean temperature profile. b) MOMIC soot volume fraction. c) Two-equations model soot volume fraction. All results include radiation effects.

From Figure 4, which shows both temperature and soot volume fraction axial profiles, it is seen that soot radiation has a large influence on flame temperature. When radiation is accounted for indeed, the maximum temperature peak has a $180 \mathrm{~K}$ decrease, from $2200 \mathrm{~K}$ to $2020 \mathrm{~K}$. Because the radiation model used here does not solve the radiative heat transfer equation, temperature variations due to soot radiation may not be adequately described. In general, because it does not consider in its formulation re-absorption, the optically thin radiation model tends to under-predict temperature peak values (Dasgupta et al., 2014). In this sense, the peak temperature predicted here (Figure 4a) may be higher than $2020 \mathrm{~K}$ and even higher than the measured experimental value. Additionally, the optically thin radiation model also tends to underpredict temperatures in most of the flame downstream region, which could be a reason for the significant temperature drop seen from $0.3 \mathrm{~m}$ above the jet exit and onwards. An important observation here is that changes in the temperature field affect the soot fields, which as a result modify the heat transfer by radiation and consequently change the temperature profile again. Additionally, it can be observed from Figure 4a that the start of the temperature drop due to radiation occurs around an axial position of $0.3 \mathrm{~m}$, which is close to the location where the soot computed peak value is found (Figure $4 \mathrm{~b}$ ).

In Figure $4 \mathrm{~b}$ the predicted values of soot volume fraction obtained with the two soot formation models studied here are compared with the experimental data of Adelaide jet flame 1 (Mahmoud et al., 2019). In the case of the two-equations model, the peak value of axial soot volume fraction (300 ppb) is higher than the experimental one (200 ppb). Regarding the MOMIC soot predictions, when employing a unity sticking factor, a soot peak value of 7 times higher than the experimental one was firstly observed. One possible explanation for this is that according to this soot model soot particles generated in the nucleation stage are modeled as spherical particles obtained from a PAH perfect coagulation and that all benzene particles that coalesce tend to form soot. It is worth noticing however that a sticking factor may be employed to decrease the nucleation rate (Blanquart and Pitsch, 2009). This enables reducing the maximum soot peak values, thus leading to results that are closer to the experimental data. For the MOMIC results shown in Figure 4b, a sticking factor of 0.07 was accounted for in the nucleation rate equation, which allowed obtaining the same peak value of $190 \mathrm{ppb}$ associated with the experiment. Additional discussions about this sticking factor are included in Section 5.2 below. 

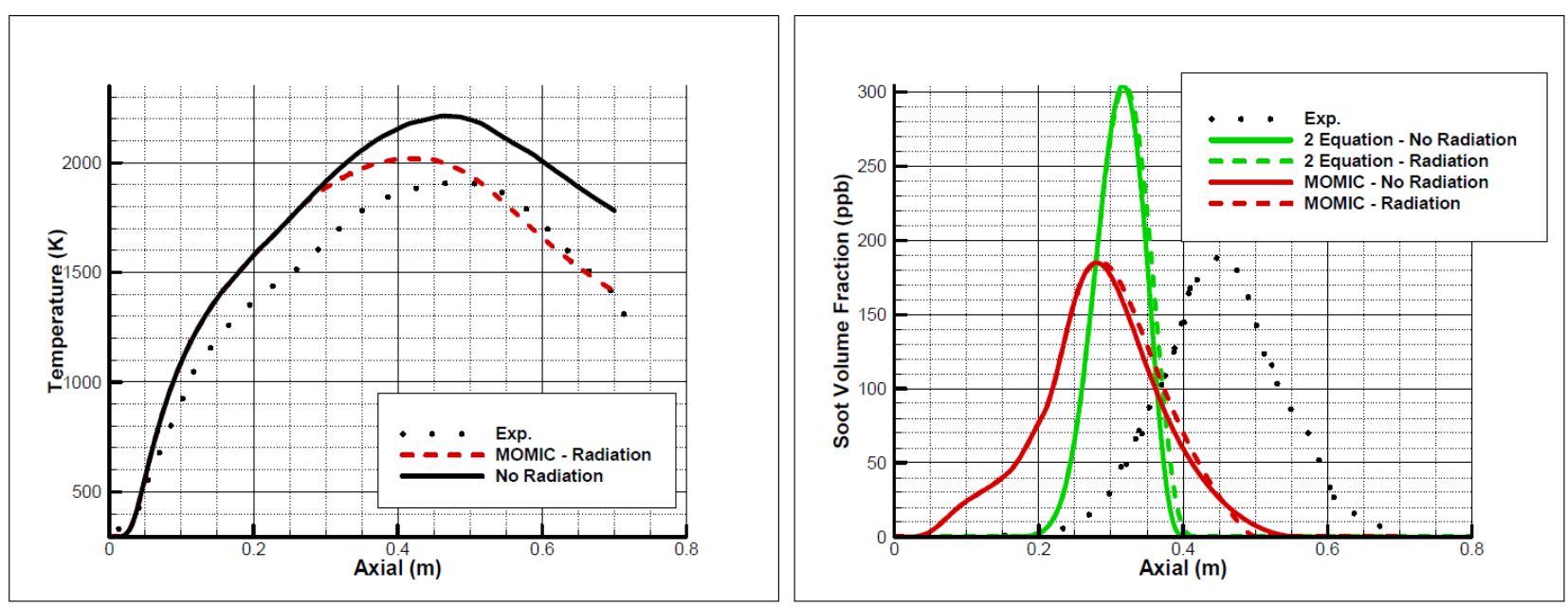

Figure 4. a) Temperature axial profiles. b) Soot volume fraction axial profiles.

\subsection{Parametric study of the MOMIC empirical constants}

Due to the fact that the MOMIC model was initially formulated for laminar premixed flames (Frenklach, 2002), the reaction rate related to the soot surface growth for reaction 4a in Appel et al. (2000) is usually modified when accounting for diffusion flame configurations. This has been done for instance in the works by Dasgupta et al. (2014), where the associated reaction rate constant is increased by factor of 2, and by Guo et al. (2004), where the same constant is increased by a factor of 5. Since the original MOMIC model (Frenklach, 2002) significantly under-predicted soot peak values, such modifications were introduced to obtain a improved agreement between the numerically predicted values and the reference experimental data. Another soot model parameter directly influencing soot predicted levels is the steric factor $\alpha$ present in the soot surface growth source term. The fitted correlation for $\alpha$ used here was the one proposed by Appel et al. (2000), which was obtained from the study of eight (8) premixed flames. Nevertheless, in several past works, a constant value for $\alpha$ has been employed to adjust the soot predicted results. Dworkin et al. (2006) used for instance a steric factor equal to 0.078, whereas Dasgupta et al. (2014) a value of 1 .

In order to assess the influence on soot predictions of the steric factor $\alpha$ present in the source terms of both surface growth and oxidation, and the sticking factor $\gamma$ present in the nucleation source term, a radially integrated soot volume fraction $\beta$, defined according to Eq. (17), was calculated in this work. The results from such computations are illustrated in Figure 5.

$$
\beta=2 \pi \int r f_{v} d r
$$
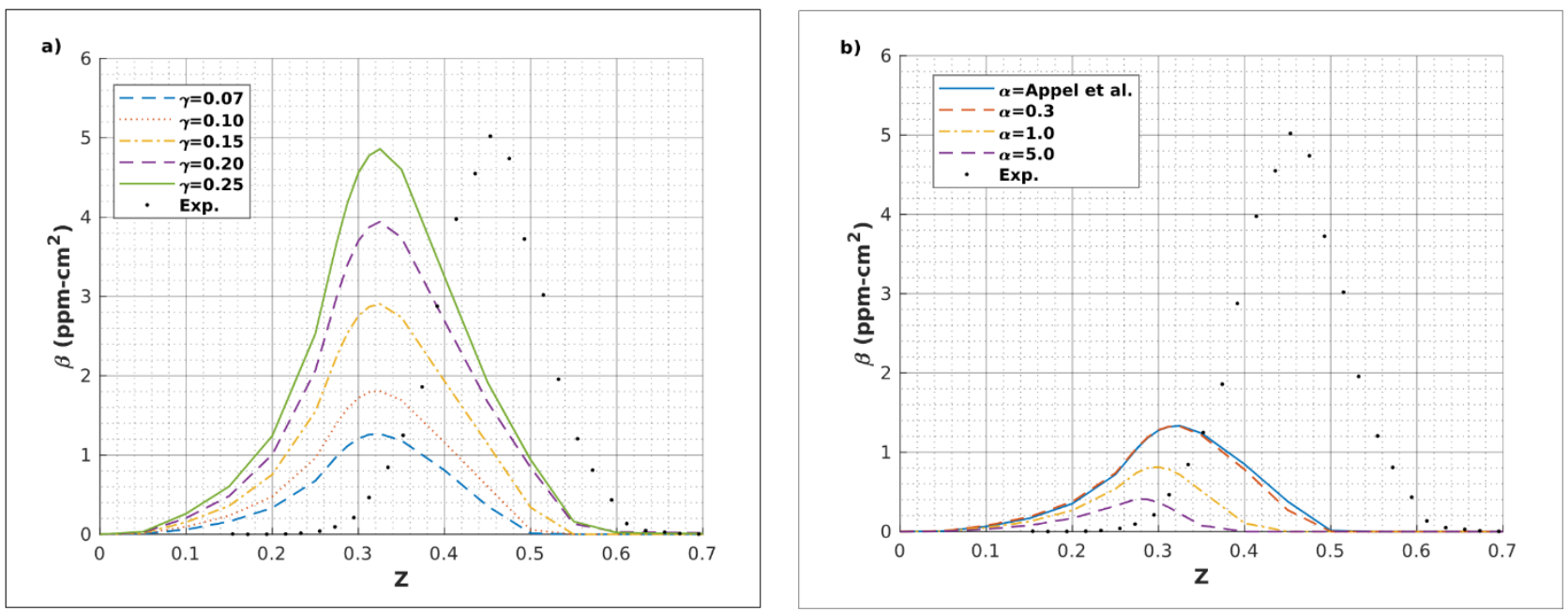

Figure 5. a) $\boldsymbol{\beta}$ axial profiles for different $\boldsymbol{\gamma}$ values. b) $\boldsymbol{\beta}$ axial profiles for different $\boldsymbol{\alpha}$ values.

Figure 5a shows $\beta$ axial profiles obtained using different values of the sticking factor $\gamma$, an empirical soot model parameter. It is noticed from this figure that the soot peak values increase with the increase in the $\gamma$ factor. No change is observed however with respect to the soot peak axial position. It is worth noticing here that the experimental peak value was reproduced by MOMIC using a $\gamma$ equal to 0.07 (Figure 4b). For this $\gamma$ value however the integrated soot profile is much lower than the experimental one (Figure 5a). This occurs because the soot radial profile obtained with the MOMIC 
model is smaller than the experimental one. In the case of the steric factor $\alpha$, Figure 5b shows that with the decrease of this factor the soot peak axial position moves downstream and the soot peak value increases. When using the $\alpha$ related correlation (Appel et al., 2000) employed here, it was observed that the average value of $\alpha$ was equal to 0.35 , so the associated result is similar to that obtained using an $\alpha$ constant and equal to 0.3. It was observed as well that when using $\alpha$ values less than 0.3 , soot profiles were unphysical. This happened because in such situations soot formation was no longer influenced by surface growth nor oxidation.

An alternative approach to shift the predicted soot profiles downstream involved changing the turbulent Schmidt number (Sct) appearing in the molecular diffusion terms present in the transport equations of mean mixture fraction $(Z)$ and mixture fraction variance (Zvar) employed in the steady laminar flamelet model (Peters, 1984). Accordingly, from Figure $6 \mathrm{~b}$ it is noticed that when increasing Sct from 0.85 to 1.2 the soot volume fraction peak shifts from 0.27 to 0.4 along the axial axis. When doing so however, the soot peak value increased by a factor of 2 , so it was necessary to adjust the sticking factor $\gamma$ to 0.03 to obtain the soot peak value of $200 \mathrm{ppb}$. Figure $6 \mathrm{a}$ also shows that the new axial temperature profile obtained with a turbulent Schmidt number equal to 1.2 reproduces relatively well the first part of the experimental data, which implies that the flame moved downstream with the Sct modification. As noticed from Figure 6a however, there is an increase in temperature in the final zone of the domain.
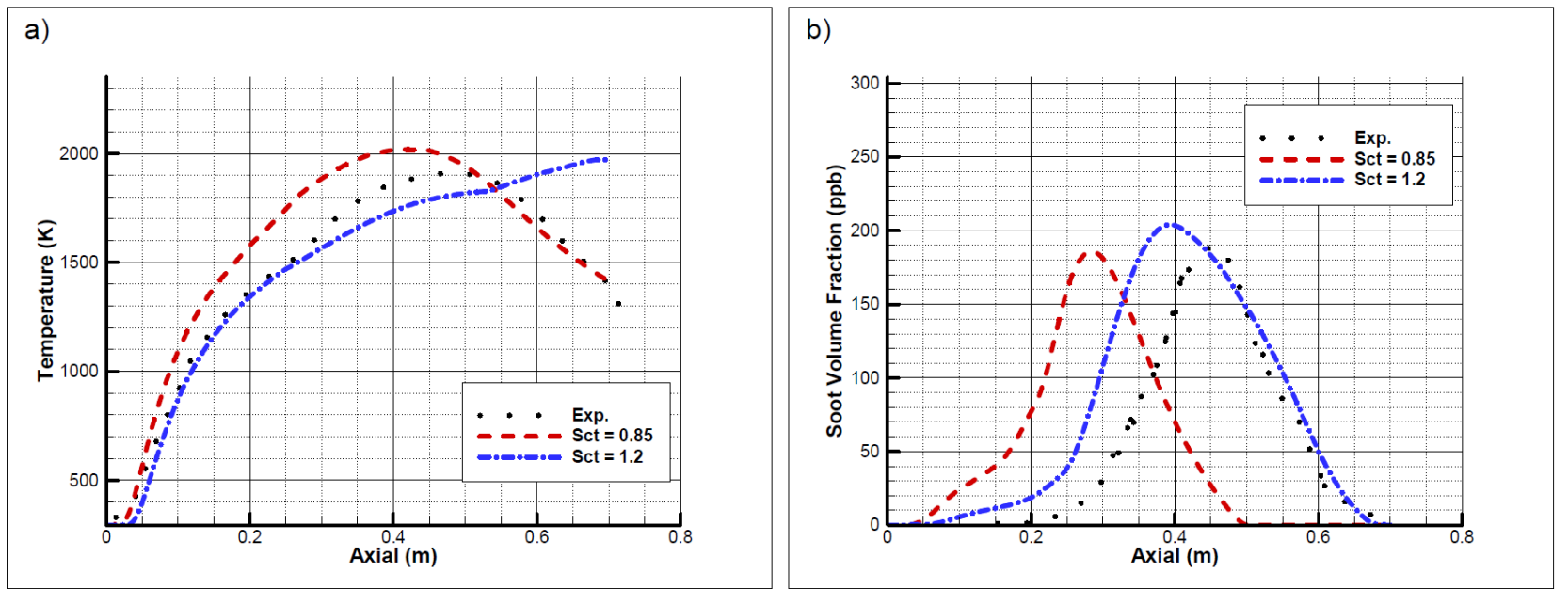

Figure 6. Turbulent Schmidt number effects on a) temperature and b) soot volume fraction axial profiles. All results include radiation effects.

\subsection{Sources of discrepancies discussion}

Another potential source of error for the relatively large amount of soot predicted by MOMIC is that related to the fact that the formation of aggregate chains is not being considered in the surface growth stage due to limitations of the model itself. In this sense, the HMOM (hybrid method of moments) model whose advantage over the MOMIC method is that it can account for a bimodal or multimodal PSDF model could be utilized (Mueller et al., 2009). In addition, the standard $k-\varepsilon$ model is also known to have limitations when used in complex flow configurations and even in axisymmetric jets with strong density changes. This turbulence model overpredicts indeed the jet spreading rate leading to a shorter flame, which can cause soot peaks to be present upstream to the point indicated by experimental results (Mahmoud et al., 2018). The way in which the fluid dynamics is solved can be therefore a large source of errors in the prediction of soot. In addition, the chemistry governing PAH evolution, which is too slow to be adequately described with the steady laminar flamelet model (Bisseti et al., 2012), represents as well another potential source of error.

Another drawback of the numerical simulations carried out here is related to use of the ABF chemical kinetic mechanism, which accounts for PAH growth via the HACA pathway mechanism (Frenklach and Wang, 1994) only. Therefore, the PAH values obtained here may have been underestimated because not all PAH growth pathways were considered. Dworkin et al. (2011) point out indeed that the HACA mechanism for PAH growth cannot adequately describe the formation of PAH species. One of the unavoidable limitations of the assessments carried out in this work is that related to the fact that no chemical species concentrations or mass fractions were measured in the experimental work used as reference here, which means that chemical species related analyses cannot not be carried out. In addition, when including radiation, predicted soot volume fractions are slightly larger than the ones when no radiation is included (Figure 3b). This may be due to the fact that soot oxidizes more quickly when it is at relatively high temperatures (Mehta et al., 2010). Generally, the effect of temperature on soot formation is difficult to predict because the chemical reactions kinetic rates increase with increasing temperature, increasing in this way not only the soot surface growth but also the oxidation of soot (Mehta et al., 2010). Finally, it is worth noticing that, in terms of quality of predictions, the numerical results obtained here are qualitatively comparable to previous ones obtained with more sophisticated models such large eddy simulation 
(LES) and transported PDF (probability density function) methods (Mueller and Raman, 2014). The soot formation models developed here are expected to be also used in future in conjunction with these high-fidelity turbulence and combustion modeling approaches.

\section{CONCLUSIONS}

A new solver for modeling soot formation is turbulent diffusion flames was developed within the OpenFOAM framework. The developed soot formation model is based on the method of moments with interpolative closure (MOMIC) and the optically thin radiation model. In order to carry out a proper coupling in terms of energy, the solver was implemented accounting for the FlameletSimpleFoam solver. Once developed, the soot model prediction accuracy was assessed trough numerical simulations of the Adelaide jet flame 1. The obtained numerical results were compared to the experimental data available in the literature, obtaining discrepancies among them in accordance with the limitations of the turbulence, combustion and soot modeling approaches employed here. The MOMIC soot model developed here attempts to describe the details of soot nucleation, coagulation, surface growth and oxidation processes. Since MOMIC based soot models were originally developed for laminar premixed flames accounting for empirical parameters, this model seems to require adaptations to properly describe soot formation in turbulent diffusion flames. As shown in this work, the soot peak values can be modified through a correct fitting of the model empirical parameters. However, the incapability of this soot model and other MOM based ones to correctly predict the axial location of the referred soot peak values is an aspect that needs to be further investigated. The results obtained here seem to suggest as well that there is no a clear advantage to switching from a semi-empirical model to a MOMIC based one. In terms of computational cost, the twoequations model solving just two additional transport equations is much more attractive because it captures a soot peak in the same order of magnitude as the experimental data. It is expected however that for different flame configurations the MOMIC soot model leads to results closer to the experimental data.

\section{ACKNOWLEDGEMENTS}

This work has been supported by CONCYTEC-FONDECYT (Peru), Contract No. 415-2019-2019-FONDECYT, "Identification of soot precursors in turbulent combustion processes through numerical modeling to reduce the impact of soot on both health and environment". During this work Luís Fernando Figueira da Silva was on leave from the Institut Pprime (CNRS - Centre National de la Recherche Scientifique, France). The authors also gratefully acknowledge the support provided by Brazil's Conselho Nacional de Desenvolvimento Cientifico e Tecnologico, CNPq, under the Research Grants No. 304444/2018-9 and 403904/2016-1.

\section{REFERENCES}

ANSYS Fluent, 2021. ANSYS Inc., https://www.ansys.com/products/fluids/ansys-fluent. Accessed 21 June 2021.

Appel, J., Bockhorn, H., and Frenklach, M., 2000. "Kinetic modeling of soot formation with detailed chemistry and physics: Laminar premixed flames of C2 hydrocarbons”. Combustion and Flame, Vol. 121, pp. 122-136.

Balthasar, M., and Kraft, M., 2003. "A stochastic approach to calculate the particle size distribution function of soot particles in laminar premixed flames”. Combustion and Flame, Vol. 133, pp. 289-298.

Balthasar, M., and Frenklach, M., 2005. "Detailed kinetic modeling of soot aggregate formation in laminar premixed flames". Combustion and Flame, Vol. 140, pp. 130-145.

Barlow, R. S., Karpetis, A. N., Frank, J. H., and Chen, J. Y., 2001. "Scalar profiles and NO formation in laminar opposedflow partially premixed methane/air flames". Combustion and Flame, Vol. 127, pp. 2102-2118.

Bisetti, F., Blanquart, G., Mueller, M. E., and Pitsch, H., 2012. "On the formation and early evolution of soot in turbulent nonpremixed flames”. Combustion and Flame, Vol. 159, pp. 317-335.

Brookes, S. J., and Moss, J. B., 1999. "Measurements of soot production and thermal radiation from confined turbulent jet diffusion flames of methane". Combustion and Flame, Vol. 116, pp. 49-61.

Blanquart, G., and Pitsch, H., 2009. "Analyzing the effects of temperature on soot formation with a joint volume-surfacehydrogen model”. Combustion and Flame, Vol. 156, pp. 1614-1626.

Cuoci, A., Frassoldati, A., Faravelli, T., and Ranzi, E., 2008. "Kinetic modeling of soot formation in turbulent non premixed flames”. Environmental Engineering Science, Vol. 25, pp. 1407-1422. 
Dasgupta, A., Roy, S., and Haworth, D. C. (2014). Detailed computational modeling of laminar and turbulent sooting flames. ACM International Conference Proceeding Series, No. 12, pp. 1-7.

Dworkin, S. B., Bennett, B. A. V, and Smooke, M. D., 2006. "A mass-conserving vorticity - velocity formulation with application to nonreacting and reacting flows”. Journal of Computational Physics, Vol. 133, pp. 430-447.

Frenklach, M., and Wang, H., 1994. "Detailed mechanism and modeling of soot particle formation". Springer Series in Chemical Physics, Vol. 59, pp. 165-192.

Frenklach, M., 2002. "Method of moments with interpolative closure”. Chemical Engineering Science, Vol, 57, pp. 22292239.

Gallen, L., Felden, A., Riber, E., and Cuenot, B., 2019. "Lagrangian tracking of soot particles in les of gas turbines". Proceedings of the Combustion Institute, Vol 37, pp. 5429-5436.

Gleason, K., Carbone, F., Sumner, A. J., Drollette, B. D., Plata, D. L., and Gomez, A., 2021. "Small aromatic hydrocarbons control the onset of soot nucleation". Combustion and Flame, Vol 223, pp. 398-406.

Guo, H., Liu, F., and Smallwood, G. J., 2004. "Soot and NO formation in counterflow ethylene/oxygen/nitrogen diffusion flames". Combustion Theory and Modelling, Vol. 8, pp. 475-489.

Holtzmann, T., 2021. Flamelet model for OpenFOAM, developed by Holtzmann CFD, available at: https://holzmanncfd.com/community/developments/creck-flamelet-model.

Kazakov, A., and Frenklach, M., 1998. "Dynamic modeling of soot particle coagulation and aggregation: Implementation with the method of moments and application to high-pressure laminar premixed flames". Combustion and Flame, Vol. 114, pp. 484-501.

Launder, B. E. and Sharma, B. I., 1974, "Application of the Energy-Dissipation Model of Turbulence to the Calculation of Flow Near a Spinning Disc", Letters in Heat and Mass Transfer, Vol. 1, No. 2, pp. 131-138.

Lee, K. B., Thring, M. W. and Beer, J. M., 1962. "On the rate of combustion of soot in a laminar soot flame". Combustion and. Flame, Vol. 6, pp. 137-145.

Lindstedt, R. P., and Louloudi, S. A., 2005. "Joint-scalar transported PDF modeling of soot formation and oxidation". Proceedings of the Combustion Institute, Vol. 30, pp. 775-783.

Mahmoud, S. M., Nathan, G. J., Alwahabi, Z. T., Sun, Z. W., Medwell, P. R., and Dally, B. B., 2018. "The effect of exit Reynolds number on soot volume fraction in turbulent non-premixed jet flames". Combustion and Flame, Vol. 187, pp. 42-51.

Mehta, R. S., Modest, M. F., and Haworth, D. C., 2010. "Radiation characteristics and turbulence-radiation interactions in sooting turbulent jet flames". Combustion Theory and Modelling, Vol. 14, pp. 105-124.

Mehta, R. S., Haworth, D. C., and Modest, M. F., 2009. “An assessment of gas-phase reaction mechanisms and soot models for laminar atmospheric-pressure ethylene-air flames". Proceedings of the Combustion Institute, Vol. 32, pp. $1327-1334$.

Mercier, X., Carrivain, O., Irimiea, C., Faccinetto, A., and Therssen, E., 2019. "Dimers of polycyclic aromatic hydrocarbons: The missing pieces in the soot formation process". Physical Chemistry Chemical Physics, Vol. 21, pp. 8285-8294.

Mueller, M. E., Blanquart, G., and Pitsch, H., 2009. "Hybrid Method of Moments for modeling soot formation and growth". Combustion and Flame, Vol. 156, pp. 1143-1155.

Mueller, M. E., and Raman, V., 2014. "Effects of turbulent combustion modeling errors on soot evolution in a turbulent non premixed jet flame". Combustion and Flame, Vol. 167, pp. 1842-1848. 
Netzell, K., Lehtiniemi, H., and Mauss, F., 2007. "Calculating the soot particle size distribution function in turbulent diffusion flames using a sectional method". Proceedings of the Combustion Institute, Vol. 31, pp. 667-674.

OpenCFD, 2021. OpenFOAM: The Open Source CFD Toolbox. OpenCFD Ltd. http://www.openfoam.com

Peters, N., 1984. "Laminar diffusion flamelet models in non-premixed turbulent combustion". Progress in Energy and Combustion Science, Vol. 10, pp. 319-339.

Pierce, C. D., and Moin, P., 2004. "Progress-variable approach for large-eddy simulation of non-premixed turbulent combustion”. Journal of Fluid Mechanics, Vol. 504, pp. 73-97.

Rezvan, K. L., N. J. Brown, and M. Frenklach, 2002. "Soot formation codes,” http://combustion.berkeley.edu/ soot/codes/codes.

Smoluchowski, M., 1917. "Mathematical theory of the kinetics of coagulation of colloidal systems". Z. Phys. Chem, Vol. 92, pp. 129-168.

Sun, Z., Dally, B., Nathan, G., \& Alwahabi, Z., 2017. "Effects of hydrogen and nitrogen on soot volume fraction, primary particle diameter and temperature in laminar ethylene/air diffusion flames". Combustion and Flame, Vol 175, pp. 270282.

Yang, S., and Mueller, M. E., 2019. “A Multi-Moment Sectional Method (MMSM) for tracking the soot Number Density Function”. Proceedings of the Combustion Institute, Vol. 37, pp. 1041-1048.

Wick, A., Frenklach, M., and Pitsch, H., 2020. "Systematic assessment of the Method of Moments with Interpolative Closure and guidelines for its application to soot particle dynamics in laminar and turbulent flames". Combustion and Flame, Vol. 214, pp. 450-463.

Zhao, H., Zheng, C., and Xu, M., 2005. "Multi-Monte Carlo method for coagulation and condensation/evaporation in dispersed systems". Journal of Colloid and Interface Science, Vol. 286, pp. 195-208.

\section{RESPONSIBILITY NOTICE}

The authors are the only responsible for the printed material included in this paper. 